\title{
Can Elimination of Epinephrine in Rhinoplasty Reduce the Side Effects: Introduction of a New Technique
}

\author{
Abdoljalil Kalantar-Hormozi • Alireza Fadaee-Naeeni • \\ Siavash Solaimanpour • Naser Mozaffari • \\ Hamed Yazdanshenas · Shahrzad Bazargan-Hejazi
}

Received: 6 September 2010/Accepted: 24 January 2011/Published online: 27 February 2011

(C) The Author(s) 2011. This article is published with open access at Springerlink.com

\begin{abstract}
Background We aim to provide evidence that despite not administering epinephrine, (1) the amount of hemorrhaging during surgery will not change, (2) surgery time will not increase and may even be shorter, and (3) there would be fewer cardiovascular-related consequences.

Methods One hundred thirteen patients were enrolled and randomized into the control $(n=74)$ and intervention groups $(n=39)$. During the primary open or closed rhinoplasty operation, anesthesia was managed by continual
\end{abstract}

A. Kalantar-Hormozi ( $\square)$

Shahid Beheshti Medical University,

15 Khordad Hospital, Tehran, Iran

e-mail: kalantarj@yahoo.com

A. Kalantar-Hormozi · N. Mozaffari

Shahid Beheshti University of Medical Sciences, Tehran, Iran

e-mail: Dr_Naser_mozafari@yahoo.com

A. Fadaee-Naeeni

Guilan University of Medical Sciences, Guilan, Iran

e-mail: fadaenaeeni@yahoo.com

S. Solaimanpour

Persian General Hospital, Tehran, Iran

e-mail: solaimanpours@yahoo.com

H. Yazdanshenas

Tehran University of Medical Sciences, Tehran, Iran

e-mail: hyazdanshenas@yahoo.com

S. Bazargan-Hejazi

Charles Drew University of Medicine and Science,

1731 E. 120th Street, Los Angeles, CA 90059, USA

e-mail: shahrzadb@ucla.edu; shahrzadbazargan@cdrewu.edu

S. Bazargan-Hejazi

Department of Psychiatry, David Geffen School of Medicine,

University of California Los Angeles, Los Angeles, CA, USA infusion of remifentanil $(14-20 \mu \mathrm{g} / \mathrm{h})$ and propofol $(4-6 \mathrm{mg} / \mathrm{kg} / \mathrm{h})$ with an infusion pump, in addition to $\mathrm{N}_{2} \mathrm{O}-\mathrm{O}_{2}(50 \%)$. Atracurium was repeated (5 mg every $20 \mathrm{~min})$. Patients in the control group received an epinephrine $(1 / 100,000)$ injection to the nose, and patients in the intervention group did not. All patients received dexamethasone ( $8 \mathrm{mg}$ IV) and metoclopramide (10 mg IV). At the end of the operation and before extubation, the muscle relaxants were reversed with prostigmine $(0.35 \mathrm{mg} / \mathrm{kg})$ and atropine $(0.175 \mathrm{mg} / \mathrm{kg})$.

Results We found (1) no statistically significant association between epinephrine injection and hemorrhage during or after surgery $(P=0.949),(2)$ a statistically significant association between epinephrine injection and complications, and (3) the group that did not receive the injection had fewer complications $(P=0.01)$. With respect to the duration of surgery, we did not detect any statistically significant associations between the groups.

Conclusion Elimination of epinephrine during rhinoplasty as an alternative procedure may lead to the same surgery outcomes if not a better one. Studies with a larger sample size are needed to further substantiate these findings.

Keywords Epinephrine $\cdot$ Lidocaine $\cdot$ Rhinoplasty $\cdot$ Local anesthetic

\section{Introduction}

With increasing demands for plastic surgery in recent years, the number of rhinoplasties has also shown an upward trend. The anatomy of the nose, with its vascular structure and limited area for maneuvering, restricts the surgeon's access and visibility during a rhinoplasty. Therefore, most surgeons have been using lidocaine/ 
epinephrine (i.e., $2 \%+1 / 100,000$; this is the combination that we refer to, for similar terms, throughout the article) with local anesthetics as a way to locally anesthetize and prepare the region for operation. Indeed, this method has become a standard procedure and current practice for most plastic surgeons [1].

Local anesthetics containing epinephrine are also routinely used in functional endoscopic sinus surgery (FESS) mainly for hemostasis [2]. In a survey of 360 otorhinolaryngologists in the UK, the majority of the surgeons reported using cocaine preoperatively because it provides a greater operative field. Nearly $70 \%$ of these surgeons reported using cocaine and epinephrine together. They considered cocaine to be safe with epinephrine more so than with lidocaine [3].

Of concern are cardiovascular side effects of epinephrine $[1,4,5]$. Sigg et al. [5] compared the hemostatic effect of a high concentration of ornipressin (5 IU/10 ml) lidocaine in patients undergoing rhinosurgery. They reported that under halothane/enflurane anesthesia, a patient's blood pressure and heart rate did not rise and remained virtually constant up to $15 \mathrm{~min}$ following local infiltration of ornipressin into the nasal tissues. However, patients who were anesthetized with diazepam/fentanyl experienced an elevation in blood pressure following infiltration of ornipressin (1-ornipressin/ epinephrine).Therefore, they recommended against the use of a high concentration (2-high concentration of mipressin/ epinephrine) for patients with blood pressure dyscrasias.

Others have warned surgeons about the rise in plasma epinephrine concentrations within minutes of epinephrine injection [6]. Hasselt et al. [6] compared plasma catecholamine concentrations after administering vasoconstrictor solutions by Moffett's method or submucosal infiltration of epinephrine $(4.4 \mathrm{ml}$ of $1: 80,000)$ and lignocaine $(2 \%)$ in 20 patients undergoing elective nasal surgery. They reported that plasma epinephrine concentrations increased by 44.3 times to a peak of $9.9 \mathrm{nmol} / 1(1,813 \mathrm{pg} / \mathrm{ml})$ within $1 \mathrm{~min}$, whereas in patients who received Moffett's solution containing epinephrine ( $1 \mathrm{ml}$ of $1: 1,000)$, the peak level of epinephrine was $1.27 \mathrm{nmol} / \mathrm{l}(232 \mathrm{pg} / \mathrm{ml})$ occurred $10 \mathrm{~min}$ after instillation of the solution $(P<0.01)$.

Cotton et al. [7] argue against the so-called "safe dose" of epinephrine (i.e., $1.0 \mathrm{~kg}^{-1}$ during halothane anesthesia). They reported that the outcomes of infiltration of lignocaine $(21 \mathrm{ml}$ of $0.5 \%)$ with epinephrine $(1: 200,000)$ to the facial area of rhinoplasty patients, and injection of lignocaine $(40 \mathrm{ml}$ of $0.5 \%)$, bupivacaine $(0.25 \%)$, and epinephrine $(1: 200,000)$ to patients undergoing brachial plexus block were different based on the site of administration. There was a $566 \%$ increase in plasma epinephrine concentration $2 \mathrm{~min}$ after cessation of injection in the rhinoplasty group, while they observed only a $112 \%$ increase in the plasma concentration of epinephrine $10 \mathrm{~min}$ after completion of the block in the brachial plexus group.
As highlighted in the above-cited literature, there is a debate among surgeons regarding the use of epinephrine with local anesthetics as a way to locally anesthetize and prepare the region for operation. This debate is partly due to the paucity of research providing convincing evidence for a safe dose of a lidocaine/epinephrine combination, especially for patients with a history of myocardial infarction or those who are sensitive to epinephrine or preservatives that are contained in lidocaine/epinephrine combination.

The overall goal of this study was to provide empirical evidence that administration of lidocaine/epinephrine with local anesthetics does not have to be considered an indispensable procedure in rhinoplasty. Specifically, we aimed to provide evidence that despite not administering lidocaine/epinephrine, (1) the amount of hemorrhaging during surgery will not change, (2) surgery time will not increase and even may be shorter, and (3) there would be fewer cardiovascular-related consequences. We hypothesized that rhinoplasty patients who do not receive lidocaine/epinephrine as a local anesthetic (case/intervention group) compared to their lidocaine/epinephrine-receiving counterparts (control group) will be less likely to (1) have extra hemorrhaging during/after surgery, (2) have prolonged surgery time, and (3) develop cardiovascular-related complications, including arrhythmia, hypertension, tachycardia, and post-surgery chest pain.

\section{Methods}

\section{Design and Procedure}

This was a randomized control pilot study conducted in Punzdahe Khordad Hospital, a plastic surgery center affiliated with Shahid Beheshti University of Medical Sciences. Patients were recruited to the study by posting study flyers in the surgery center from May 2008 to May 2009. Interested patients who called in were screened by the study coordinator. If eligible and still interested, an initial appointment for the informed consent procedure, further screening, and collection of baseline information and measurements was scheduled. To be eligible for the study, male and female patients had to meet all the following inclusion criteria: (1) be 18 years old or older, (2) scheduled for a primary open or closed rhinoplasty operation, (3) have no history of or current cardiovascular diseases and disorders, (4) have normal values for all the preoperation laboratory test results (PT, PTT, and INR), and (5) willing to sign an informed consent form. Patients who were participating in another study and/or could not meet the aforementioned criteria were excluded from the study. The rest of the study procedures were conducted in three stages. 


\section{First Stage: Screening}

During a scheduled initial visit, the following procedures were conducted to find out if the subjects met all the study requirements and if it was safe for them to be involved in the study. To do so, the study coordinator once more screened potential subjects for participation eligibility and explained the research protocol and all its details to them. Patients were given ample amount of time to review the informed consent form and were given an opportunity to ask questions of the coordinator and/or physicians who were involved in the trial. Only after all of the patients' questions and concerns were answered did they sign and date a written informed consent form. Only patients who met all the study eligibility requirements and signed he informed consent form were entered into the study and were scheduled for the baseline assessment. The principle of the study protocol was approved by the ethics committee of Shahid Beheshti University of Medical Sciences.

\section{Second Stage: Baseline}

During the baseline visit, all enrolled patients received a physical examination, and preoperation tests and subsequent information were recorded. They also filled out a baseline patient information sheet, including their contact information; number of physician-diagnosed diseases, specifically cardiovascular diseases; past operations; allergies; and medication history, including number and types of any over-the-counter or physician-prescribed medications. Again, all patients with any indication of cardiovascular diseases were removed from the study.

\section{Third Stage: Randomization}

In this stage participating patients were randomly assigned (using pre-group-assigned sealed envelopes) to either the intervention group $(n=39)$ or the control group/standard care $(n=74)$ using computerized random number allocation.

\section{Measurements}

The study outcome variables include hemorrhage recorded as $<50 \mathrm{cc}$, between 50 and $100 \mathrm{cc}$, and $>100 \mathrm{cc}$; surgery duration recorded in minutes; and post-surgery temporary cardiovascular complications measured as any sign of arrhythmia (yes, no) and hypertension. Hypertension was measured as minimum vs. maximum blood pressure (BP), where patients with systolic $\leq 160$ and diastolic $\leq 80$ were grouped in the normal BP category and those with systolic $>160$ and diastolic $>80$ were grouped in the abnormal BP category. Other variables in the study include gender (male, female), age (18 and older), weight ( $\mathrm{kg})$, and pulse rate (PR) (beats/min).

\section{Rhinoplasty Procedures}

All the operations (primary open and closed rhinoplasties) were performed using the standard technique/protocol without any changes. The steps to perform open rhinoplasty were carried out in the following order: Typically, the operation began by giving general anesthesia. This was followed by the injection of anesthetic solution (lidocaine/ epinephrine), and finally making a columellar incision. The steps to perform closed rhinoplasty were first administering general anesthesia, followed by injection of anesthetic solution (lidocaine/epinephrine), and finally making an intercartilaginous incision.

Of note, however, is that for the patients in the intervention group, the second step in both the open and the closed rhinoplasty was eliminated (i.e., they did not receive the standard dose of lidocaine/epinephrine injection during the surgery), and we made the incision right after general anesthesia. Patients in the standard care group did receive the standard dose of adrenalin injection before starting surgery.

As is known, septoplasty can prolong the duration of the rhinoplasty operation. To eliminate the threat of procedure bias, patients who needed a septoplasty were equally divided between cases and control groups.

\section{Intervention}

At first, all patients were premedicated with midazolam (1 mg IV) and remifentanil (1-1.5 $\mu \mathrm{g} / \mathrm{kg})$. Then they were anesthetized with nesdonal $(5 \mathrm{mg} / \mathrm{kg})$ and atracurium $(0.6 \mathrm{mg} / \mathrm{kg})$ as muscle relaxants. After $2 \mathrm{~min}$, patients were intubated with an appropriate tracheal tube and the cutoff tube was filled with air and its pressure became constant at $25 \mathrm{cmH}_{2} \mathrm{O}$.

Following the injection, the septal flap was elevated and the submucosal area was resected. The nasal tip operation was performed by transcartilage incision. Finally, the dorsal hump was flattened by rasp and cartilage excision using scissors and a blade, and osteotomy was also performed.

Anesthesia was managed by continual infusion of remifentanil $(14-20 \mu \mathrm{g} / \mathrm{h})$ and propofol $(4-6 \mathrm{mg} / \mathrm{kg} / \mathrm{h})$ with an infusion pump, in addition to $\mathrm{N}_{2} \mathrm{O}-\mathrm{O}_{2}(50 \%)$. Atracurium was repeated (5 $\mathrm{mg}$ every $20 \mathrm{~min}$ ). Then epinephrine was injected into the nose of patients in the standard care group (control group), but patients in the intervention group did not receive this injection. All patients received dexamethasone ( $8 \mathrm{mg} \mathrm{IV}$ ) and metoclopramide (10 mg IV).

At the end of the operation and before extubation, muscle relaxants were reversed with prostigmine 
$(0.35 \mathrm{mg} / \mathrm{kg})$ and atropine $(0.175 \mathrm{mg} / \mathrm{kg})$. Except for the elimination of the lidocaine/epinephrine injection for the intervention group, all procedures used during the operation were considered standard measures or techniques for any rhinoplasty surgery. During and after the operation, exact recordings of possible arrhythmias, blood pressure fluctuations, and pulse rate variations were registered in both the intervention and the standard care group. Moreover, all patients were asked if they were suffering from cardiac symptoms (i.e., chest pain) after the operation. Recovery time after the operation, the amount of bleeding, and patient satisfaction were also recorded.

\section{Data Analysis}

Univariate analysis using descriptive statistics (i.e., frequency, percentage, mean and standard deviation) was used to present distributions of the main variables in the study. Independent sample $t$ tests and $\chi^{2}$ tests of association were used, when appropriate, to evaluate differences in the main outcome variables (i.e., hemorrhage, surgery duration, post-surgery temporary cardiovascular complications) between the intervention and the standard care group. A $P$ value of less than 0.05 was considered statistically significant. Data were analyzed using the SPSS ver. 16.0 (SPSS Inc., Chicago, IL).

\section{Results}

The main purpose of this study was to demonstrate that administration of epinephrine with local anesthetics does not have to be considered an indispensable procedure in rhinoplasty operations. One hundred thirteen patients were enrolled in the study and randomized into the control/ standard care group $(n=74)$ and the intervention group $(n=39)$. The majority of patients were female $(87.6 \%)$. Participants' mean age and standard deviation was $25.4 \pm 7.3$ years. No statistically significant associations were detected between the intervention group and the standard care group with respect to the baseline characteristics (age, gender, weight, BP) (Table 1).

We hypothesized that patients without injection of epinephrine (the intervention group) will have less hemorrhaging, fewer complications, and a shorter surgery time. Results of the $\chi^{2}$ test of associations indicated no statistically significant association between epinephrine injection and hemorrhage during or after surgery $(P=0.949)$. Although more complications were recorded for patients in the standard care group who received epinephrine (i.e., one patient with arrhythmia, one patient with HTN, and three patients with both complications) than in patients in the intervention group for whom no complications were
Table 1 Overall characteristics of the sample $(n=113)$

\begin{tabular}{|c|c|}
\hline Variable & Frequency $(\%)$ \\
\hline Adrenalin injection & $74(65.5)$ \\
\hline \multicolumn{2}{|l|}{ Sex } \\
\hline Male & $14(12.4)$ \\
\hline Female & 99 (87.6) \\
\hline Epinephrine not injected & $39(34.5)$ \\
\hline \multicolumn{2}{|l|}{ Hemorrhage } \\
\hline$<50 \mathrm{cc}$ & $72(63.7)$ \\
\hline $50-100 \mathrm{cc}$ & $31(27.4)$ \\
\hline$>100 \mathrm{cc}$ & $10(8.8)$ \\
\hline \multicolumn{2}{|l|}{ Complications } \\
\hline Arrhythmia & $1(0.9)$ \\
\hline Hypertension & $1(0.9)$ \\
\hline None & $108(95.6)$ \\
\hline Both & $3(2.7)$ \\
\hline \multicolumn{2}{|l|}{ Procedure } \\
\hline Rhino closed (primary) & $83(73.4)$ \\
\hline \multirow[t]{2}{*}{ Septo Rhino open (primary) } & $30(26.6)$ \\
\hline & Mean $\pm \mathrm{SD}$ \\
\hline Age & {$[25.5 \pm 7.3]$} \\
\hline Weight & {$[59.9 \pm 10.6]$} \\
\hline BP min & {$[53.5 \pm 6.5]$} \\
\hline BP max & {$[76.1 \pm 9.3]$} \\
\hline Pulse rate & {$[71.8 \pm 5.4]$} \\
\hline $\mathrm{PCO}_{2}$ & {$[22.1 \pm 2.06]$} \\
\hline $\mathrm{O}_{2}$ sat & {$[97.7 \pm .79]$} \\
\hline Time & {$[126.1 \pm 28.7]$} \\
\hline
\end{tabular}

recorded, the association was not statistically significant $(P=0.161)$. With respect to the duration of surgery, using a $t$ test, the average surgery time for the intervention group was recorded as (mean $\pm \mathrm{SD}$ ) $113.7 \pm 22.6 \mathrm{~min}$ compared with the standard care group whose operation time was $149.8 \pm 23.9 \mathrm{~min}$; the difference between the two groups was statistically significant $(P=0.001)$ (Table 2$)$.

\section{Discussion}

There has been an upward trend in the number of rhinoplasties in recent years. Also, more surgeons are using epinephrine during the surgery. This study evaluated whether elimination of epinephrine during the operation would make any difference in the amount of bleeding during and after surgery, the length of the operation, and cardiovascular-related complications, including arrhythmia, hypertension, tachycardia, and post-surgery chest pain. Our findings showed that elimination of epinephrine in fact significantly shortened the length of surgery from 
Table 2 Association between epinephrine, sex, age, hemorrhage, and complications across the intervention and control groups

\begin{tabular}{|c|c|c|c|c|}
\hline Variable & $\begin{array}{l}\text { Control group } \\
\text { with epinephrine } \\
(N=74) \\
F(\%)\end{array}$ & $\begin{array}{l}\text { Intervention group } \\
\text { without epinephrine } \\
(N=39) \\
F(\%)\end{array}$ & $\chi$ & $P$ \\
\hline Sex & & & 6.26 & 0.016 \\
\hline Male & $5(6.8)$ & $9(23.0)$ & & \\
\hline Female & $69(93.2)$ & $30(77.0)$ & & \\
\hline Hemorrhage & & & 0.104 & 0.949 \\
\hline$<50 \mathrm{cc}$ & $47(63.5)$ & $25(64.1)$ & & \\
\hline $50-100 \mathrm{cc}$ & $20(27.0)$ & $11(28.2)$ & & \\
\hline$>100 \mathrm{cc}$ & $7(9.5)$ & $3(7.7)$ & & \\
\hline Complications & & & 9.92 & 0.019 \\
\hline Arrhythmia & $1(2.6)$ & $0(0.0)$ & & \\
\hline Hypertension & $1(2.5)$ & $0(0.0)$ & & \\
\hline None & $74(100.0)$ & $34(87.1)$ & & \\
\hline Both & $0(0.0)$ & $3(7.7)$ & & \\
\hline Age $[N, \mathrm{M} \pm \mathrm{SD}, t(\mathrm{df})]$ & {$[74,25.7 \pm 7.3,0.581(111)]$} & {$[39,24.9 \pm 7.3,0.583(77)]$} & & 0.562 \\
\hline Duration $[N, \mathrm{M} \pm \mathrm{SD}, t(\mathrm{df})]$ & {$[74,149.8 \pm 22.6,7.88(111)]$} & {$[39,113.7 \pm 23.9,7.75(74)]$} & & 0.001 \\
\hline
\end{tabular}

149 min (average length of surgery in the control group) to 113.7 min (average length of surgery in the intervention group) $(P=0.001)$; reduced the number of expected complications in the intervention group, even though this association was not statistically significant; and did not add an additional risk of bleeding.

Our evidence raises the possibility that elimination of epinephrine during rhinoplasty as an alternative procedure may in fact lead to the same surgery outcome if not better. Indeed, we observed fewer complications among a few patients who did not receive epinephrine. This finding adds to the existing concerns regarding the use of epinephrine to locally numb the area of operation during rhinoplasty. Previous studies have suggested that during rhinoplasty injection of epinephrine was more likely to elevate the patient's blood pressure, thus raising the risk of cardiovascular-related side effects. Koeppe et al. [1] argued that injection of even a very small dose of epinephrine as a local anesthetic can increase plasma catecholamines. In their study, overall cardiovascular-related side effects of prilocaine and lidocaine were reported at $5.9 \%$ of rhinoplasties and $8.1 \%$ of face-lifts, percentages that they believed were quite high for such procedures. They strongly suggested that surgeons should use ropivacaine more often since it offers significant advantages in both efficacy and prolonged duration of analgesia. According to their findings, use of ropivacaine also reduces the risk for adverse side effects due to less toxicity.

Demirtas et al. [4] studied the hemodynamic effects of lidocaine/epinephrine in healthy patients who underwent rhinoplasty procedures. They concluded that after the injection of these medications, a mild to moderate and short-lasting tachycardia was detected. There was also an increase in the pulse rate during lateral osteotomies.

John et al. [8] reported that all patients showed a marked increase in plasma epinephrine concentration within 4 min of injecting epinephrine 1:80,000 and 2\% lignocaine. Therefore, they warn surgeons to be aware of this marked but unpredictable systemic absorption of locally infiltrated vasoconstrictors during any functional endoscopic sinus surgeries.

In a preliminary study, Yang et al. [2] reported that among FESS patients, epinephrine $(1: 200,000)$ contained in $2 \%$ lidocaine or saline did cause temporary hypotension and other hemodynamic changes that lasted approximately 4 min. Surgeons have also been cautioned about the possibilities of drug interactions with the lidocaine/epinephrine combination [9].

In the current study, there was less of a chance of cardiac issues by not using epinephrine; there were two complications in the control group: one patient with hypertension and one with an arrhythmia. Even though this was not found to be significant, not using epinephrine did not add any additional cardiac complications and did not increase the amount of bleeding in the intervention group. Nonetheless, elimination of epinephrine did significantly reduce the duration of surgery and we did observe a trend toward fewer complications in the intervention group.

\section{Conclusion}

This study was a randomized control pilot study. Patients were randomized to either the intervention group (only 
lidocaine injection) or the control group (lidocaine with epinephrine). We aimed to show that epinephrine can be avoided in the local anesthetic solution used for rhinoplasty.

Our results indicate that elimination of epinephrine for a few patients in the intervention group reduced the number of anticipated cardiac-related complications. Furthermore, elimination of epinephrine did not add any additional risk of bleeding assessed intraoperatively by sponge count and total aspirate. Our findings also showed that elimination of epinephrine reduced the length of surgery in the intervention group, which could be partly due to the 5-10 min saved by not having to inject the lidocaine/epinephrine. These findings raise the possibility that elimination of epinephrine during the rhinoplasty could be an alternative procedure that may in fact lead to the same surgery outcome if not a better one. Studies with a larger sample size can help further substantiate these findings.

Acknowledgments The authors are grateful to the staff of Punzdahe Khordad Hospital, a plastic surgery center affiliated with Shahid Beheshti University of Medical Sciences, for their rigorous collaboration in patient recruitment and data collection. The project was entirely funded by a grant from Shahid Beheshti University of Medical Sciences.

Disclosure The authors have no conflicts of interest or financial ties to disclose.

Open Access This article is distributed under the terms of the Creative Commons Attribution Noncommercial License which permits any noncommercial use, distribution, and reproduction in any medium, provided the original author(s) and source are credited.

\section{References}

1. Koeppe T, Constantinescu MA, Schneider J, Gubisch W (2005) Current trends in local anesthesia in cosmetic plastic surgery of the head and neck: results of a German national survey and observations on the use of ropivacaine. Plast Reconstr Surg 115(6):1723-1730

2. Yang JJ, Li WY, Jil Q, Wang ZY, Sun J, Wang QP, Li ZQ, Xu JG (2005) Local anesthesia for functional endoscopic sinus surgery employing small volumes of epinephrine-containing solutions of lidocaine produces profound hypotension. Acta Anaesthesiol Scand 49(10):1471-1476

3. De R, Uppal HS, Shehab ZP, Hilger AW, Wilson PS, CourteneyHarris R (2003) Current practices of cocaine administration by UK otorhinolaryngologists. J Laryngol Otol 117(2):109-112

4. Demirtas Y, Ayhan S, Tulmac M, Findikcioglu F, Ozkose Z, Yalcin R, Atabay K (2005) Hemodynamic effects of perioperative stressor events during rhinoplasty. Plast Reconstr Surg 115(2):620-626

5. Sigg O, Pirsig W, Hirlinger WK (1983) Ornipressin as vasoconstrictor in rhinosurgery. Rhinology 21(2):159-164

6. van Hasselt CA, Low JM, Waldron J, Gibb AG, Oh TE (1992) Plasma catecholamine levels following topical application versus infiltration of epinephrine for nasal surgery. Anaesth Intensive Care 20(3):332-336

7. Cotton BR, Henderson HP, Achola KJ, Smith G (1986) Changes in plasma catecholamine concentrations following infiltration with large volumes of local anaesthetic solution containing epinephrine. Br J Anaesth 58(6):593-597

8. John G, Low JM, Tan PE, van Hasselt CA (1995) Plasma catecholamine levels during functional endoscopic sinus surgery. Clin Otolaryngol Allied Sci 20(3):213-215

9. Asaadi M, Anagnoste SR (1995) The use of cold normal saline for vasoconstriction in rhinoplasty. Plast Reconstr Surg 96(3):751-752 